\title{
Uterus Didelphys with Obstructed Hemivagina and Ipsilateral Renal Agenesis (OHVIRA Syndrome)
}

\author{
Mauri José Piazza ${ }^{1}$, Newton Sergio De Carvalhoํㄱ ${ }^{1}$ Ana Paula Lisboa Peixoto ${ }^{1}$, Almir Antonio Urbanetz ${ }^{1}$ \\ ${ }^{1}$ Gynecology and Obstetrics Department. Federal University of Paraná - Gynecology Endocrinology Sector, \\ Curitiba, PR, Brazil
}

\begin{abstract}
This paper aimed to report a series of 19 cases of uterus didelphys associated with obstructed hemivagina and ipsilateral renal agenesis. This retrospective descriptive observational study included the medical records of 19 patients seen at the Endocrinology Gynecology Sector of the Obstetrics and Gynecology Department of the Federal University of Paraná, and focused on clinical data, complementary exams, patient management, and follow-up. From the 229 patients with genital anomalies seen between 1984 and $2009,19(8.3 \%)$ were diagnosed with uterus didelphys with vaginal septum and renal agenesis. The patients had a median age of \pm 16.3 years. Eight subjects $(42.6 \%)$ reported abdominal pain; two of them $(0.1 \%)$ had pelvic masses and seven (36.8\%) had dysmenorrhea. Blood retention was confirmed by ultrasound and/ or magnetic resonance imaging of the pelvis, which revealed the existence of a duplicated uterus of the didelphys type with a cystic mass containing old blood obliterating the hemivagina. Urinary tract examination revealed the existence of ipsilateral renal agenesis. Unusual manifestations such as associated infection and rupture of the vaginal septum during sexual intercourse were also reported. The prevalence of uterus didelphys associated with obstructed hemivagina and ipsilateral renal agenesis was $8.3 \%$ in a series of 229 genital anomalies. The observance of clinical features is essential for the early identification of the syndrome and the adequate management of the patients.
\end{abstract}

Keywords: Urogenital abnormalities, Mullerian ducts, Didelphys abnormalities, Uterus congenital defects

\section{INTRODUCTION}

The syndrome of uterus didelphys with oblique vaginal septum and ipsilateral renal agenesis is a rare Mullerian anomaly. It is believed to occur around the $8^{\text {th }}$ week of gestation when the Mullerian ducts and the adjacent mesonephric duct are simultaneously affected. This formation defect results in a unilaterally obstructed vaginal septum with menstrual flux accumulation proximal to the obstruction. Usually difficult to diagnose when asymptomatic, this anomaly should be contemplated in young women with abdominal pain and/or dysmenorrhea and/or presenting abdominal mass due to retention of menstrual blood with a consequent hematocolpos and hematometra.

Clinical presentation is characterized by dysmenorrhea and pelvic pain, shortly followed by menarche and possibly associated with the presence of vaginal or pelvic mass. In addition to clinical examination, imaging methods (ultrasonography or magnetic resonance) are used to confirm the uterine malformation and the associated renal anomaly. The aim of the treatment is to eliminate the menstrual retention through the surgical resection of the vaginal septum.

The present study comprehends the analysis of data from 19 patients with uterus didelphys, oblique vaginal septum with obstructed hemivagina with hematocolpos, and ipsilateral renal agenesis (Rock \& Jones, 1980), suffer- ing from a condition known as Herlyn-Werner-Wunderlich syndrome (Wunderlich, 1976) or obstructed hemivagina and ipsilateral renal anomaly (OHVIRA) syndrome (Herlyn \& Werner, 1971; Smith \& Laufer, 2007). Early and accurate diagnosis is of the utmost importance to avoid complications and maintain the reproductive capacity of the affected patients.

The purpose of this study was to describe the clinical findings, the related signs and symptoms, complementary imaging methods, and the diagnosis and management of the patients with uterus didelphys with obstructed hemivagina and ipsilateral renal agenesis seen at the Endocrinology Gynecology sector of the Clinics Hospital from the Federal University of Paraná between 1994 and 2009, and to review the literature on this malformation. Patient clinical data, complementary exams, treatment, and follow-up are presented, and the prevalence of the malformation among the 229 genital malformations found in the sector is evaluated.

\section{MATERIAL AND METHODS}

This retrospective descriptive observational study was based on the collection of data on Mullerian malformations described in patient medical records. The study was approved by the Human Research Ethics Committee of the Clinics Hospital from the Federal University of Paraná (UFPR) and complied with GPC and Brazilian regulations. A protocol to manage the clinical records was first designed considering clinical history data, complementary exams, and patient management according to each diagnosed condition. In the 25-year period covered by the study (from 1984 to 2009), nineteen of the 229 patients with genital malformations seen at the Gynecology Endocrinology Sector of the Federal University of Paraná were diagnosed with this anomaly.

Inclusion criteria: patients with clinical data and clinical findings confirmed by both gynecological physical examination and imaging, categorized into Group III of the nomenclature established by the American Fertility Society / American Society of Reproductive Medicine (AFS / ASRM) (The American Fertility Society 1988), or into Group U3-V2-Bicorporeal complete uterus and longitudinal obstructed vaginal septum as per the ESHRE/ESGE Classification (Grimbizis et al., 2013)

Exclusion criteria: other contexts of menstrual retention and/or similar malformations e.g. unicornuate uterus, cavitated rudimentary uterine horn. Robert's syndrome, a usually cited similar uterine malformation, was not found in this study.

\section{RESULTS}

Nineteen of the 229 patients seen for uterovaginal anomalies from 1994 to 2009 met the study's inclusion criteria. The included patients had a mean age of \pm 16.3 years. At the time of diagnosis, the youngest patient was 12 years old, and the oldest was 19 .

Abdominal pain and dysmenorrhea were the main symptoms reported by 15 of the 19 patients, and two developed 


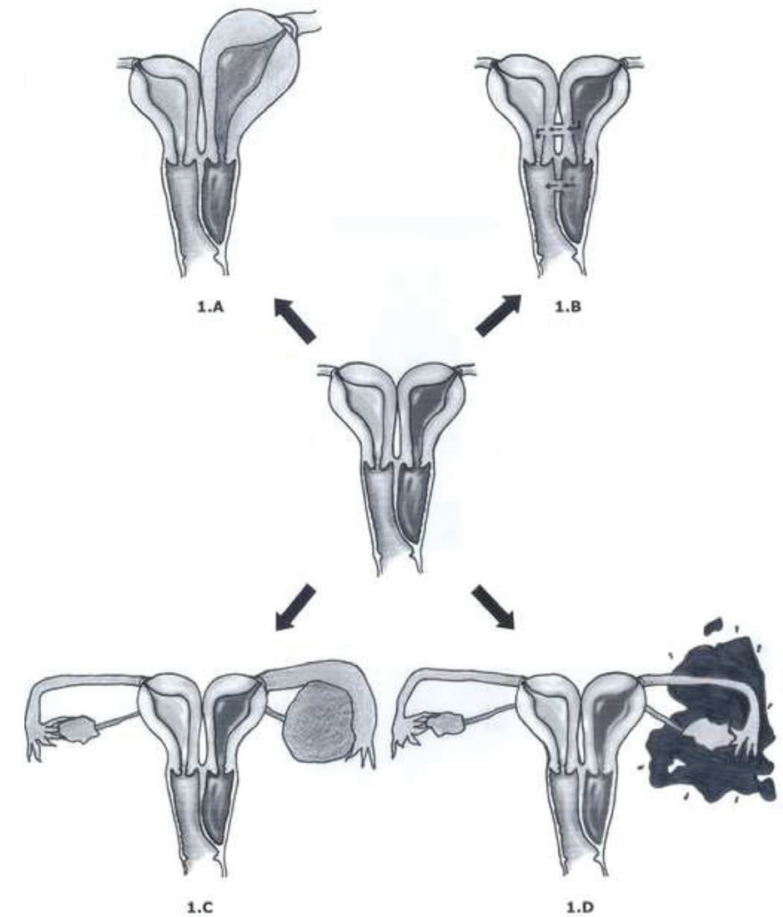

Figure 1 - schematic representation of uterus didelphys with left oblique vaginal septum and hematocolpos adapted from Gidwani \& Falcone (1999) with approval. $A, B, C$ and $D$ - Clinical feature variations according to the different levels of blood retention and complications Figure $1 \mathrm{~A}$ - blood retention with associated tumor formation

Figure $1 \mathrm{~B}$ - discrete menstrual discharge through a small puncture

Figure $1 \mathrm{C}$ - tubo-ovarian abscess - TOA (specific case with a $1000 \mathrm{~cm}^{3}$ TOA)

Figure $1 \mathrm{D}$ - blood draining into the peritoneum (increasing the chance of endometriosis)

associated tumors (Figure $1 \mathrm{~A}$ ) due to progressive menstrual blood accumulation. Abdominal palpation revealed the presence of a mass, and patients had hematocolpos and hematometra. In two patients complaining of scarce menses a small puncture was observed at the level of the vaginal obstruction to the septum with very little menstrual blood (Figure $1 \mathrm{~B}$ ). Signs and symptoms of genital infection were found in two other subjects due to contamination of the retained blood by vaginal microorganisms; one had fever and the other a $1000 \mathrm{~cm}^{3}$ tube ovarian abscess seen in ultrasound examination (Figure $1 \mathrm{C}$ ). The remaining two patients were asymptomatic and were diagnosed based on 1) vaginal examination and observation of the vaginal mass (hematocolpos), and 2) after profuse bleeding caused by rupture of the hematocolpos following vaginal intercourse. Clinical examination of the latter patient revealed a lacerated left oblique vaginal septum. Ultrasound imaging showed the individual had left renal agenesis and uterus didelphys. Figure 1D shows the onset of endometriosis as a possible consequence of blood retention in the uterine cavity and the subsequent flow of blood into the peritoneum.

All patients underwent physical and gynecological examination and the most significant finding was an obstructed hemivagina by an oblique vaginal septum and the presence of a lump in the lateral vaginal wall with hematocolpos. In nine patients, the vaginal septum obstructed the right hemivagina and in ten subjects the occlusion was on the left side. Complete uterine duplication of the uterus didelphys type (Figure 2) was defined with the aid of ultrasound and/or magnetic resonance imaging of the pelvis. Renal agenesis ipsilateral to the vaginal septum was identified after simultaneous urinary tract ultrasound imaging and/or excretory urography. In this sample, right renal agenesis was observed in nine patients and left renal agenesis in ten subjects. All patients were treated with resection of the vaginal septum and drainage of the hematocolpos. One of them had a pelvic infectious complication, which was defined as acute pelvic inflammatory disease after the procedure. All these finding are described in Table 1.

Late follow-up post-operative examination revealed patients were normal and free of anomalies.

\section{DISCUSSION}

The actual incidence of reproductive system anomalies is unknown, but occurrence has been reported to vary between $0.1 \%$ and $0.38 \%$ in females (Burgis, 2001). This variation is certainly due to poor accuracy of diagnostic methods and the great diversity of possible clinical manifestations.

The literature on the described syndrome is scarce and the main findings derive from reports of isolated cases (Gholoum et al., 2006; Zurawin et al., 2004; Haddad et al., 1999). The exact etiology of this malformation is not entirely known, but the simultaneous occurrence of malformations in the Mullerian and Wolffian ducts has been considered. The association between uterovaginal and renal malformations is frequent as the Wolffian ducts play a role in the formation of the kidneys and the internal genitalia (Gholoum et al., 2006; Zurawin et al., 2004; Vercellini et al., 2007; Coskun et al., 2008; Acien et al., 2009; Prada Arias et al., 2005). Genital anomalies may have their origin either in a failure in the fusion of the Mullerian ducts in the midline and in their failure in connecting with the urogenital sinus, or in a failure in the canalization between the

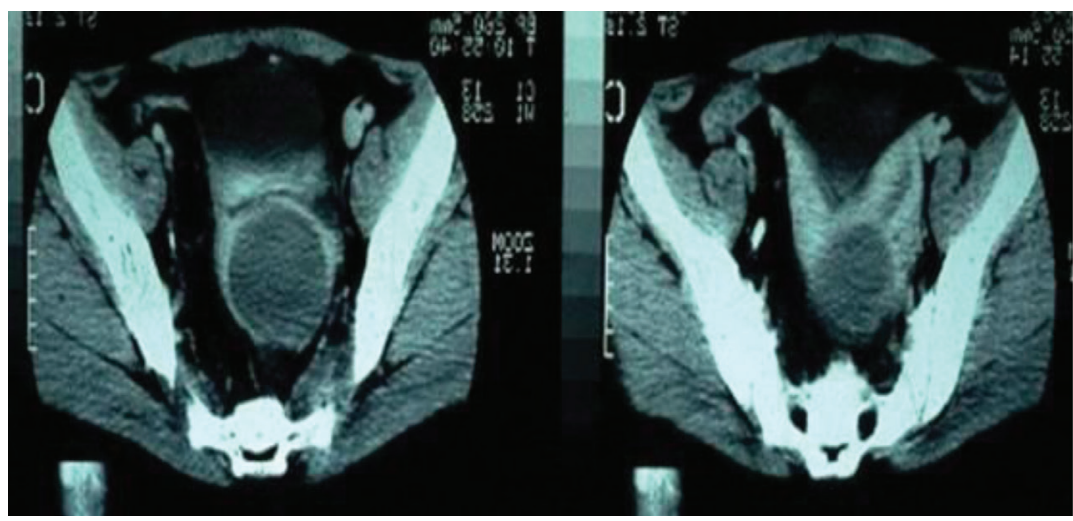

Figure 2 - Magnetic resonance imaging of the pelvis showing uterus didelphys and a large mass of the hematocolpos type in the left hemivagina.

(Case 12 on Table 1) 
Table 1- Clinical findings

\begin{tabular}{|c|c|c|c|}
\hline Case & Age & Complaints & Examination (Gynecologic/Ultrasound/MRI) \\
\hline 1 & 14 & Abdominal pain & Right vaginal septum/right renal agenesis \\
\hline 2 & 15 & Pain/vaginal tumor & Hematocolpos/right vag. septum/right renal agenesis \\
\hline 3 & 29 & Vaginal Cyst & Right vaginal septum/right renal agenesis \\
\hline 4 & 15 & Abdominal pain & Left vaginal septum/left renal agenesis \\
\hline 5 & 14 & Menstrual pain & Right vaginal septum/right renal agenesis \\
\hline 6 & 15 & Menstrual pain & Left vaginal septum/left renal agenesis \\
\hline 7 & 22 & Menstrual pain & Right vaginal septum/right renal agenesis \\
\hline 8 & 16 & Menstrual pain & Right vaginal septum/right renal agenesis \\
\hline 9 & 15 & Abdominal pain/mass & Left vaginal hematocolpos/left renal agenesis \\
\hline 10 & 16 & Abdominal pain & Left vaginal septum/hematocolpos/left renal agenesis \\
\hline 11 & 16 & Menstrual pain & Left vaginal septum/left renal agenesis \\
\hline 12 & 12 & Abdominal pain & Left vaginal septum/left renal agenesis \\
\hline 13 & 15 & Abdominal pain & Right vaginal septum/right renal agenesis \\
\hline 14 & 13 & Menstrual pain & Right vaginal septum/right renal agenesis \\
\hline 15 & 13 & Abdominal pain & Left vaginal septum/left renal agenesis \\
\hline 16 & 14 & Vaginal mass & Right vaginal septum/right renal agenesis \\
\hline 17 & 15 & Pain/vaginal mass & Left vaginal septum/hematocolpos/left renal agenesis \\
\hline 18 & 14 & Menstrual pain & Left vaginal septum/left renal agenesis \\
\hline 19 & 18 & Post-coital bleeding & Laceration Left vaginal septum/left renal agenesis \\
\hline
\end{tabular}

superior portion of the vagina and the uterus.

Uterus didelphys occurs due to a failure in the fusion between both Mullerian ducts resulting in the duplication of the uterine body, the canal, and also possibly of the vagina. A vaginal septum is present in $75 \%$ of the cases of uterus didelphys. This entity is usually suspected in clinical manifestations appearing during puberty or menarche. Affected patients often complain of recurrent progressive pelvic pain caused either by hemivagina dilatation in the occurrence of hematocolpos, by the dilatation of the uterine cavity in cases of hematometra, or by the accumulation of blood in the uterine tubes with the occurrence of hematosalpinx. Blood stasis and retrograde menstruation of the obstructive syndrome may also lead to blood drainage into the peritoneal cavity (hemoperitoneum) as in Figure $1 \mathrm{D}$. All these findings were evidenced in this study, with the main clinical manifestation being the occurrence of pelvic pain and dysmenorrhea. The intermittent retrograde blood flow seen in this type of syndrome may influence the development of pelvic endometriosis and bring severe subsequent consequences including fertility reduction (Batt \& Mitwally, 2003; Sanfilippo et al., 1986; Hur et al., 2007; Singh et al., 2009). While reviewing the literature on 102 superficial malformations (Zurawin et al., 2004; Haddad et al., 1999), an asymmetric distribution between affected and unaffected sides of the body was observed - right hemibody involvement was seen in 57/102 of the cases, while left hemibody anomalies were reported in 37/102 of the subjects; the distribution was equitable in the remaining six cases reported. Preferential hemibody involvement was not observed in the 19 cases studied in our series, as nine patients had right hemibody and ten had left hemibody involvement.

Most Mullerian malformations - e.g. septate or bicornuate uterus - are symmetrically distributed, and lateral asymmetry is less frequently observed in cases of uterus didelphys with obstructing hemivaginal septum and unilat- eral renal agenesis (Smith \& Laufer, 2007). In our study, ten patients had a vaginal septum obstructing the left hemivagina and nine subjects had obstructed right hemivagina, suggesting that the syndrome does not preferentially involve one of the sides of the body. A study carried out more than 30 years ago (Rock \& Jones, 1980) noted a more common occurrence of obstructed hemivagina and renal agenesis on the right side, as also observed more recently by other authors (Vercellini et al., 2007). Although this is not a common anomaly, prognosis is excellent when patients are diagnosed early on and adequately managed. Early detection, surgical removal of the vaginal septum, and vaginoplasty alleviate symptoms and prevent the consequences of chronic obstruction such as endometriosis, pelvic adherences, and infectious collections (pyocolpos, pyometrium, and pyosalpinx (Wu et al., 2007) and even hydronephrosis). (Al Kadri et al.,2009) Definitive treatment requires the surgical excision of the septum and the drainage of the blood in the obstructed vagina as early as possible. It is very important to pay close attention to the differential diagnosis versus other malformations that may also induce menstrual blood retention, such as unicornuate uterus with an appendix situated laterally and containing a responsive endometrium, or Robert's uterus. It should also be mentioned that the asymptomatic patient (profuse bleeding caused by the rupture of the hematocolpos following vaginal intercourse) in this series seems to be a rather unique case.

Fertility in women with uterus didelphys is not notably impaired and prognosis is comparatively good, while prematurity and fetal growth retardation indicate the necessity of meticulous prenatal care (Heinonen, 2000).

\section{CONCLUSION}

In this study, the analysis of clinical data from medical records showed that 19 patients had a syndrome comprised of uterus didelphys, oblique septum, and re- 
nal agenesis ipsilateral to the septum. Considering the low number of cases described in the literature, this series may help increase the relevance of the data reported to date and inform the management of similar cases. Diagnosis in early adolescence provides for improved management and helps prevent the complications of menstrual blood retention and the concomitant pain symptoms.

\section{CONFLICT OF INTERESTS}

No conflict of interest have been declared.

\section{Corresponding author:}

Mauri José Piazza

Gynecology and Obstetrics Department. Federal University of Paraná

Gynecology Endocrinology Sector, Curitiba, PR, Brazil

E-mail: mauripiazza@hotmail.com

\section{REFERENCES}

Acien $\mathrm{P}$, Acien M, Sanchez-Ferrer ML. Mullerian anomalies "without a classification": from the didelphys-unicollis uterus to the bicervical uterus with or without septate vagina. Fertil Steril. 2009; 91:2369-75.

Al Kadri H, Al-Hunain S, Rubaish S, Alamir A, Alfakeeh K. Didelphic uterus and obstructed hemivagina resulting in obstructed hydronephrosis of transplanted kidney. J Pediatr Surg 2009; 44:e13-5.

Batt RE, Mitwally MF. Endometriosis from telarche to midteens: pathogenesis and prognosis, prevention and pedagogy. J Pediatr Adolesc Gynecol. 2003;16:337-47.

Burgis J. Obstructive Mullerian anomalies: case report, diagnosis and management. Am J Obstet Gynecol 2001; 185:338-44

Coskun A, Okur N, Ozdemir O, Kiran G, Arykan DC. Uterus didelphys with an obstructed unilateral vagina by transverse vaginal septum associated with ipsilateral renal agenesis, duplication of inferior vena cava, high-riding aortic bifurcation, and intestinal malrotation: a case report. Fertil Steril. 2008; 90:2006.e9-11.

Gholoum S, Puligandla PS, Hui T, Su W, Quiros E, Laberge JM. Management and outcome of patients with combined vaginal septum, bifid uterus, and ipsilateral renal agenesis (Herlyn-Werner-Wunderlich syndrome). J Pediatr Surg. 2006; 41:987-92.

Gidwani G, Falcone T, eds. Congenital malformations of the female genital tract: diagnosis and management. Lippincott Williams \& Wilkins; Philadelphia: 1999.

Grimbizis GF, Gordts S, Di Spiezio Sardo A, Brucker S, De Angelis C, Gergolet M, Li TC, Tanos V, Brölmann H, Gianaroli L, Campo R. The ESHRE/ESGE consensus on the classification of female genital tract congenital anomalies. Human Reprod. 2013; 28:2032-44.

Haddad B, Barranger E, Paniel. Blind hemivagina: longterm follow-up and reproductive performance in 42 cases. Human Reprod. 1999; 14:1962-4.
Heinonen PK. Clinical implication of the didelphic uterus: long-term follow-up of 49 cases. Eur J Obstet Gynecol Reprod Biol. 2000;91:183-90.

Herlyn U, Werner H. [Simultaneous occurrence of an open Gartner-duct cyst, a homolateral aplasia of the kidney and a double uterus as a typical syndrome of abnormalities]. Geburtshilfe Frauenheilkd. 1971; 31: 340-7.

Hur JY, Shin JH, Lee JK, Oh MJ, Saw HS, Park YK, Lee KW. Septate uterus with double cervices, unilaterally obstructed vaginal septum, and ipsilateral renal agenesis: a rare combination of Mullerian and Wolffian anomalies complicated by severe endometriosis in an adolescent. J Minim Invasive Gynecol. 2007;14:128-31.

Prada Arias M, Muguerza Vellibre $R$, Montero Sánchez $M$, Vázquez Castelo JL, Arias González M, Rodríguez Costa A. Uterus didelphys with obstructed hemivagina and multicystic dysplastic kidney. Eur J Pediatr Surg. 2005;15:441-5.

Rock JA, Jones HW Jr. The double uterus associated with an obstructed hemivagina and ipsilateral renal agenesis. Am J Obstet Gynecol 1980;138:339-42.

Sanfilippo JS, Wakim NG, Schikler KN, Yussman MA. Endometriosis in association with uterine anomaly. Am J Obstet Gynecol. 1986;154:39-43.

Singh L, Bosemani $\mathrm{T}$, Brandom $\mathrm{H}$, Hughes $\mathrm{T}$. Uterus didelphys and obstructed right hemivagina associated with ipsilateral ovarian endometriosis and renal agenesis. Eur J Radiol Extra 2009; e131-5.

Smith NA, Laufer MR. Obstructed hemivagina and ipsilateral renal anomaly (OHVIRA) syndrome management and follow-up. Fertil Steril. 2007; 87:918-22.

The American Fertility Society. Classifications of adnexal adhesions, distal tubal occlusion, tubal occlusion secondary to tubal ligation, tubal pregnancies, Mullerian anomalies and intrauterine adhesions. Fertil Steril. 1988; 49:944-55,

Vercellini $P$, Daguati $R$, Somigliana $E$, Viganò $P$, Lanzani $A$, Fedele $\mathrm{L}$. Asymetric lateral distribution of obstructed hemivagina and renal agenesis in women with uterus didelphys: institutional case series and a systematic literature review. Fertil Steril. 2007; 87:719-24.

Wunderlich M. [Unusual form of genital malformation with aplasia of the right kidney]. Zentralbl Gynakol. 1976;98:559-62.

Wu WC, Chang WC, Yeh LS, Yang TC. Didelphic uterus and obstructive hemivagina with ipsilateral renal agenesis complicated by pyocolpos. Taiwan J Obstet Gynecol. 2007; $46: 295-8$.

Zurawin RK, Dietrich JE, Heard MJ, Edwards CL. Didelphic uterus and obstructed hemivagina with renal agenesis: case report and review of the literature. J Pediatr Adolesc Gynecol. 2004; 17:137-41. 